\title{
Post traumatic osteoma of tibial insertion of medial collateral ligament of knee joint
}

\author{
V Shiv Shanker, S Gadikoppula, M D Loeffler
}

\begin{abstract}
Two cases are presented of post traumatic para-articular osteoma developing at the site of tibial attachment of the medial collateral ligament of knee joint. These occurred after injuries sustained while playing football and in one case the ossified mass was treated with surgical excision for unresolved symptoms after conservative measures. A comparison is made with Pellegrini Stieda disease, which is a similar affection of the femoral insertion of the medial ligament of the knee joint.

(BrF Sports Med 1998;32:73-74)
\end{abstract}

Keywords: post traumatic osteoma; medial collateral ligament; knee

The development of new bone in the region of femoral attachment of medial collateral ligament of knee after injury was described by Kohler in $1903,{ }^{1}$ but it is better known as Pellegrini Stieda disease or syndrome. ${ }^{12}$ However, a similar condition involving the tibial insertion of medial collateral ligament is less well recognised. In this report we present the case histories of two patients who sustained injury to the medial collateral ligament and later developed ossification at the site of tibial attachment.

\section{Case No 1}

A 17 year old footballer was injured in a block tackle in October 1995. At the time of impact his right knee was short of full extension and in extreme lateral rotation. He was examined by his team physiotherapist who detected laxity of medial ligament and a moderate anterior draw of his knee.

After an initial period of rest in a brace, he was treated with ultrasound and short wave diathermy as well as non-weight bearing quadriceps exercises. However, he experienced momentary painful giving way and occasional clicks in his right knee particularly when attempting to play.

When examined in the clinic in February 1996, he had a painful lump $5 \mathrm{~cm}$ below the joint line on the medial aspect of the knee joint. There was no laxity of the medial ligament but slightly increased anterior laxity compared with his left knee. There was no effusion or joint line tenderness.

Radiographs of the right knee (fig 1) showed a small bipartite mass at the site of tibial insertion of medial collateral ligament without any soft tissue abnormality or periosteal reaction.

In view of recurrent episodes of painful giving way, surgery was carried out. Arthroscopy showed a stable joint without any intraarticular abnormality. Excision of the lump was performed through a longitudinal incision. Histopathological examination showed reactive bone merging with collagenous fibrous tissue. There was osteoblastic and osteoclastic activity without any evidence of neoplasm.

Postoperative rehabilitation was supervised by a physiotherapist and at the time of final review in May 1996, there was complete resolution of pain and the patient had returned to competitive football.

\section{Case No 2}

A 26 year old man sustained a valgus injury to his left knee while playing soccer in January 1996. Since then he complained of episodes of pain and swelling on the medial aspect of the knee particularly after attempting to play soccer. There was no history of locking or giving way. Examination showed tenderness over the tibial insertion of the medial ligament with normal overlying soft tissue. Valgus stress

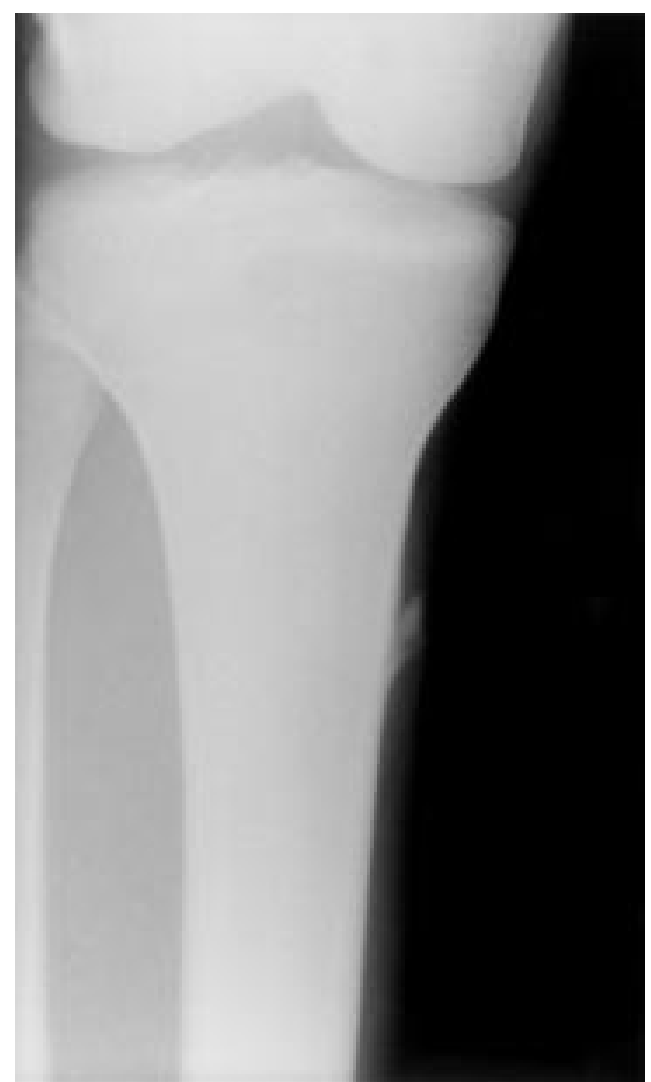

Figure 1 Anteroposterior radiograph of the right knee showing a small bipartite bony density arising from the tibial shaft in the region of attachment of the collateral ligament. 


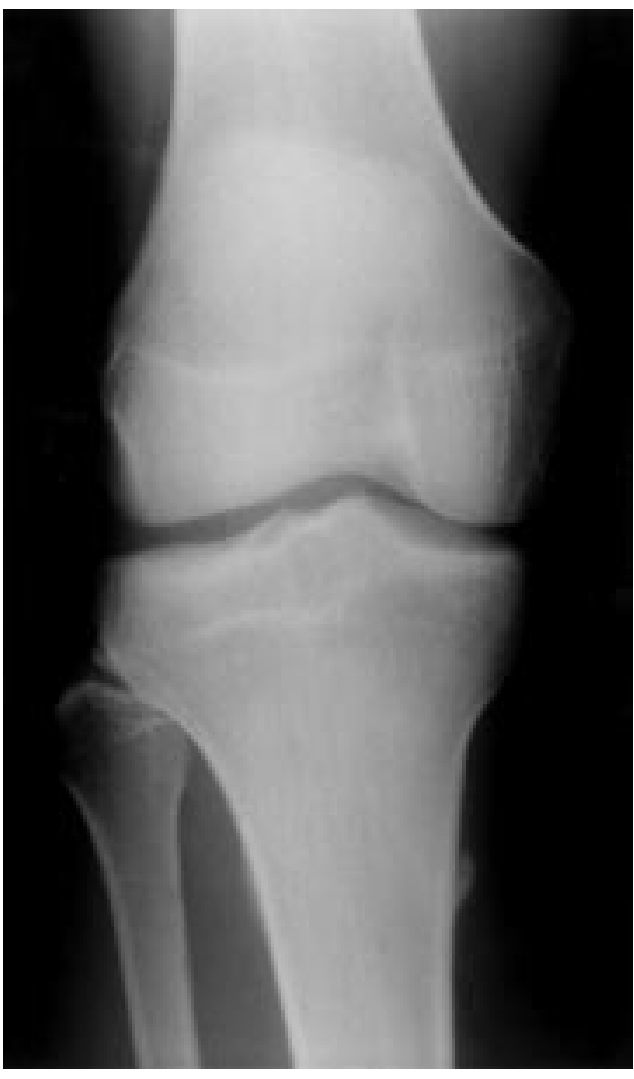

Figure 2 There is a dense ossified mass arising at the site of the tibial insertion of the medial collateral ligament.

caused increased pain but there was no instability. There was no effusion or joint line tenderness.

Radiography (fig 2) showed a dense opacity at the site of tibial attachment of the medial collateral ligament extending into the body of the ligament.

The patient was advised to refrain from contact sports and any activity that aggravated his symptoms for a period of 10 weeks. This resulted in resolution of tenderness and he was allowed gradual resumption of training and sporting activity. At the time of last review, 14 months after initial injury, he remained asymptomatic.

\section{Discussion}

Para-articular ossification after trauma in the region of proximal medial collateral ligament of knee is a well recognised entity and carries the eponym Pellegrini Stieda disease ${ }^{1}$ or syndrome. ${ }^{2}$ The involvement of the tibial insertion of this ligament in post traumatic ossification is less well recognised. A diligent search of English medical literature did not yield any reference to such a condition.

In both the cases reported here, there was a definite trauma sustained, resulting in valgus injury to the medial collateral ligament. The condition evolved as an avulsion sprain of the medial ligament, which did not respond to physiotherapy. The essential feature was activity related pain, which could be localised as tenderness over the tibial attachment of the medial ligament. In neither of the cases was there soft tissue inflammation or joint effusion. In contrast Pellegrini Stieda disease is associated with painful limitation of flexion ${ }^{3}$ and this can mimic septic arthritis. ${ }^{4}$

Histopathological examination of the excised lesion in the first case showed cartilage as well as reactive osteoblasts and osteoclasts. This is similar to the findings in Pellegrini Stieda disease as described by Kulowski.

The diagnosis is aided by radiographic examination, though it is possible that, as in Pellegrini Stieda disease, early cases would show abnormal 99m technetium MDP uptake, before the evolution of plain radiographic changes. ${ }^{6}$ Post traumatic osteoma has to be distinguished from solitary congenital exostosis of the proximal tibia. An exostosis (osteochondroma), would demonstrate cortical and cancellous bone in continuity with the parent bone and would typically point away from the nearby joint and towards the diaphysis.

Well defined and sharply marginated excrescences in relation to ligamentous and tendinous insertion after trauma represent traction injury. Bony outgrowths are also a known feature of non-traumatic conditions. Degenerative joint disorders can present marginal or capsular osteophytes, which are lips of bone resulting from endochondral ossification at the non-pressure segments of joint. ${ }^{8}$ Degenerative spondylosis with segmental instability, is associated with traction osteophytes, which develope in relation to the outer fibres of annulus fibrosus. Enthesopathy, with erosion and prolifertion at the site of osseous attachment of ligaments and tendons, is a characterstic feature of seronegative spondyloarthritis. ${ }^{9}$ Post traumatic osteoma occur in isolation, and any nearby joints do not show the clinical and radiological features of the degenerative and inflammatory joint disorders.

When dealing with post traumatic osteomas, an expectant line of management with avoidance of aggravating activity and physiotherapy should be instituted initially. However, as in our first case, if a painful lump persists despite adequate conservative measures surgical excision of the mass can be undertaken. In both our cases, there was complete resolution of the symptoms with full return to sporting activities.

1 Bentley G. Affections of the knee joint. In : Duthie RB, Bentley G, ed. Mercer's orthopaedic surgery. London: Arnold, 1996;1183-4.

2 Wang JC, Shapiro MS. Pellegrini - Stieda Syndrome. Am $\mathcal{F}$ Orthop 1995;24:493-7.

3 Trickey EL. Injuries of the knee. In: Wilson JN, ed. Watsonfones fractures and joint injury. London: Longman Group, 1982:1035.

4 Scheib JS, Quinet RJ. Pellegrini - Stieda syndrome mimicking acute septic arthritis. South Med f 1989;82:90-1.

5 Kulowski J. Pellegrini - Stieda's disease. FAMA 1933;100:

114-17.
6 Ren-Shyan Liu, Chrong-Sonq Chou, Shin-Hwa Yeh. Clin Nucl Med 1987;12:47-9.

7 Resnick D, Kyriakos M, Greenway GD. Tumors and tumorlike lesions of bone: imaging and pathology of specific lesions. In: Resnick D, ed. Diagnosis of bone and joint disorlesions. In: Resnick D, ed. Diagnosis of bone

ders. Philadelphia: Saunders, 1995: 3278 .
8 Resnick D, Niwayama. Degenarative disease of extraspinal Resnick D, Niwayama. Degenarative disease of extraspinal
location.In : Resnick D, ed. Diagnosis of bone and joint disorlocation.In : Resnick D, ed. Diagnosis of bone and
ders. Philadelphia: Saunders, 1995: 1278-81.

9 Resnick D, Niwayama. Rheumatoid arthritis and seronegative spondyloarthropathies : radiographic and pathologic concepts. In: Resnick D, ed. Diagnosis of bone and joint disorders. Philadelphia: Saunders, 1995: 860. 